\title{
A Practical Approach for 4G Systems: Deployment of Overlay Networks
}

\author{
Pablo Vidales, Glenford Mapp, Frank Stajano, Jon Crowcroft \\ Computer Laboratory, University of Cambridge \\ William Gates Building, 15 JJ Thomson Avenue, Cambridge, UK \\ \{pav25, gem11, fms27, jac22\}@cam.ac.uk \\ Carlos Jesús Bernardos \\ University Carlos III of Madrid \\ Avda. Universidad 30, Leganés, Madrid, Spain \\ cjbc@it.uc3m.es
}

\begin{abstract}
Experimental activities play a vital role in the deployment and development of novel radio access networks. In particular the movement from $3 G$ to $4 G$ poses new challenges, which need to be solved using practical approaches such as testbeds. This paper presents a testbed that can be regarded as an early attempt to build a $4 G$ system. It fully integrates heterogeneous wireless technologies using a loosely-coupled architecture. Also, experimental results are included to show the possibilities of this setup.
\end{abstract}

\section{Introduction}

We are witnessing the development and deployment of a large number of wireless networking technologies including 3G, WLANs, Bluetooth, and Ultrawideband. At the same time we are seeing a convergence of core networking infrastructure on the Internet Protocol Suite (IP) [19]. IPv4 is widely deployed throughout the Internet and there is now a serious effort to deploy IPv6, which simplifies mobility support.

Thus, there is a significant need to propose a single unified approach that integrates all disparate wireless technologies (see Table 1), and enables mobile terminals to seamlessly roam between access networks while they enjoy a plethora of IP-based services. This convergence poses many challenges, which need to be solved before the deployment of a real $4 \mathrm{G}$ network.

Inter-technology handovers (i.e. changing connection between two access points from different radio access technologies, also called vertical handovers) are challenging to current transport protocols, because packets get lost or delayed during the handover affecting overall performance.
Moreover, methods to minimise latency during vertical handovers need to be developed in order to support real time applications in these future systems.

\begin{tabular}{|l||l|l|l|}
\hline Network & Coverage & Data Rates & Cost \\
\hline \hline Satellite & World & Max. 144 kb/s & High \\
\hline GSM/GPRS & Aprox. 35 Km & $9.6 \mathrm{~kb} / \mathrm{s}$ up to $144 \mathrm{~kb} / \mathrm{s}$ & High \\
\hline IEEE 802.16a & Aprox. 30 Km & Max. $70 \mathrm{Mb} / \mathrm{s}$ & Medium \\
\hline IEEE 802.20 & Aprox. $20 \mathrm{Km}$ & $1-9 \mathrm{Mb} / \mathrm{s}$ & High \\
\hline UMTS & $20 \mathrm{Km}$ & up to $2 \mathrm{Mb} / \mathrm{s}$ & High \\
\hline HIPERLAN 2 & 70 up to $300 \mathrm{~m}$ & $25 \mathrm{Mb} / \mathrm{s}$ & Low \\
\hline IEEE 802.11a & 50 up to $300 \mathrm{~m}$ & $54 \mathrm{Mb} / \mathrm{s}$ & Low \\
\hline IEEE 802.11b & 50 up to $300 \mathrm{~m}$ & $11 \mathrm{Mb} / \mathrm{s}$ & Low \\
\hline Bluetooth & $10 \mathrm{~m}$ & Max. $700 \mathrm{~kb} / \mathrm{s}$ & Low \\
\hline
\end{tabular}

Table 1. Diversity in existing and emerging wireless technologies.

Mobility management and terminal location are other challenges that need to be solved before the challenge of ubiquitous connectivity is addressed. IP does not support mobility, as it was designed for fixed networks. Moreover, present commercial protocols are inadequate in dealing with vertical handovers. Thus, we need to include support for location management and seamless roaming in today's protocol stack. An early step in this direction is Mobile IP, which has been developed to support mobility. Mobile IPv4 [28] has been deployed for sometime while Mobile IPv6 has just recently been made an RFC [20]. However,we believe that better mobility management schemes are needed to support this new environment.

In 2002, the Laboratory for Communication Engineering (LCE) and the Computer Laboratory (CL) at the University of Cambridge came together to develop a MIPv6-based testbed (LCE-CL testbed), which will be used in order to study these issues. This paper presents the design, integra- 
tion and development of the testbed. The goal of these efforts was to produce a platform that fully integrates heterogeneous wireless technologies anticipating that in the near future mobile devices will have several wireless interfaces and users will expect connections to be seamlessly managed. In that sense, the testbed can be regarded as a prototype of a $4 \mathrm{G}$ system, focused on the mobility aspects.

The paper is structured as follows: Section 2 introduces the operation of the Mobile IPv6 protocol. Section 3 compares the LCE-CL testbed with previous projects, focusing on the aspects that make it useful for the deployment of 4G. Section 4 looks at the architecture selected to integrate the wireless networks. Section 5 introduces the technologies that support the MIPv6-based platform, mentioning the most important aspects. Section 6 describes the experimental setup, showing a network-centric view. The software components are described in Section 7. Finally, Section 8 summarises some experimental results gathered in previous research activities, and we conclude in Section 9.

\section{Mobile IPv6 basic operation}

Mobile IPv6 [21] specifies two main scenarios. First, when the Mobile Node (i.e. mobile terminal) is connected to its Home Network (where the mobile device is registered as a local entity), the packets between the correspondent nodes (i.e. the nodes communicating with the Mobile Node) and the Mobile Node are delivered using normal IP routing. Second, when the MN is connected to a foreign network (not connected to its home network), a bidirectional tunnel is created between the Mobile Node (MN) and the Home Agent (HA), which is a special router that supports packet routing to the registered nodes. The HA encapsulates the packets and routes them to the MN using its Care-of Address (CoA), assigned to the terminal by a local authority in the visited network.

MIPv6 also defines a route optimisation procedure to avoid the triangle routing problem caused by the use of bidirectional tunnelling. This mechanism basically enables the MNs to send Binding Update (BU) messages also to the Correspondent Node $(\mathrm{CN})$, not only to the HA, in order to inform about the MN's current location (i.e. MN's CoA). Once the $\mathrm{CN}$ is aware of the actual location of the $\mathrm{MN}$, the communication is direct between $\mathrm{CN}$ and $\mathrm{MN}$, bypassing the HA.

\section{Comparison with previous work}

Several testbeds have been proposed (and some of them implemented) that emulate 4G systems. Most of these environments provide limited mobility between heterogeneous networks based on existing mobility management protocols (e.g., MIPv4, MIPv6, and SIP). Below we compare some previous testbeds, based on Mobile IP, with our work.

The concepts of wireless overlay networks and vertical handover were introduced in 1996, as part of the BARWAN project at Berkeley $[22,32]$. The first overlay networks testbed, the BARWAN testbed, included WaveLAN, Infrared, and Ricochet wireless networks. Obviously, this testbed was based on MIPv4 and it was the pioneer project in the area of mobile networking.

Other researchers proposed many testbeds and simulations [12, 35], concentrating on the evaluation of MIPv4 during intra-technology handovers (i.e. horizontal handovers), for example, the Stanford testbed, MosquitoNet [24].

Later on, with the growth of IPv6 and the deployment of MIPv6, a new generation of testbeds appeared. Now, with novel protocols, researchers concentrate on minimising delays during horizontal handovers and some of them focus on evaluating MIPv6 performance in heterogeneous environments.

In 2002, EURESCOM [3] funded an European testbed $[16,17]$ to evaluate the use of Mobile IP in an All-IP core network. At the end of this project [31], they implemented a MIPv4-based testbed that integrated GPRS, LAN, and WLAN as sample technologies, and they evaluated MIPv4 and Cellular IP as mobility management protocols. However, this project focused on recommendations and they did not published practical results related to handover issues. The LCE-CL testbed was entirely evaluated and this performance study was presented in [13].

MIPv6-based testbeds were used to study the integration of different radio access technologies into one IP-based core infrastructure. A recent example, Moby Dick [14, 25] proposed and implemented a global end-to-end MIPv6-based architecture to offer QoS in heterogeneous environments. The testbed included UMTS-like TD-CDMA wireless access technology, IEEE 802.11b WLANs, and wired connectivity. Further work is being done as part of a new initiative: Daidalos project [2]. Nevertheless, the lack of access to a real operator's $3 \mathrm{G}$ network is the main difference between these projects and the LCE-CL setup.

The Nomad [5] project terminated in June 2004 [29], and it successfully set up a MIPv4-based testbed [23, 15], whereas the LCE-CL is based on MIPv6. While they evaluated seamless roaming between heterogeneous networks based on MIPv4 -assuming the presence of foreign agents in each visited network -, they did not analyse the performance of MIPv6 in 4G networks. MIPv6 is a better candidate for mobility in future networks as it has been optimised accordingly to the demands imposed by mobility management in integrated networks - foreign agents have been eliminated by the use of protocols such as IPv6 stateless or stateful (DHCPv6) autoconfiguration. 


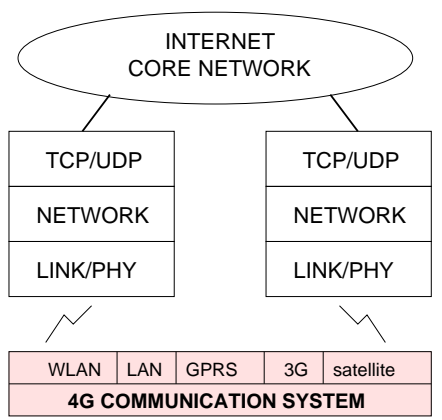

A. Tunneled Networks Model

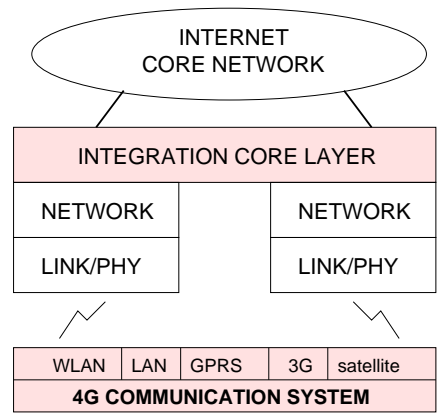

B. Hybrid Networks Model
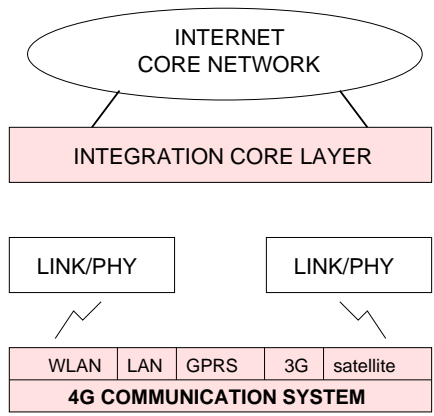

C. Heterogeneous Networks Model

Figure 1. Taxonomy according to the integration layer.

MIND [4] is the follow up of the IST project BRAIN (Broadband Radio Access for IP based Networks). These two projects implemented an experimental setup, which integrated IEEE 802.b, UMTS TDD, and GPRS. They evaluated MIPL during inter- and intra-technology handovers $[18,30]$. However, due to the impossibility of MIND to have access to the operator's infrastructure, there are two important deficiencies in comparison with our testbed. First, there is no integration between the networks (i.e. open coupling), which implies duplicity in all the processes, adding overhead during Mobile Node's roaming. Second, the mobile nodes are behind a NAT (Network Address Translator), this means that direct IPv6 over IPv4 tunnelling cannot be used.

\section{Integrated Networks Taxonomy}

One of the main features of the $4 \mathrm{G}$ communication systems is the inter-operation of multiple radio access technologies (RATs). Contrary to homogeneous environments, many approaches can be taken depending on the level of integration between different RATs - and this integration is correlated with the degree of modification to each individual technology. This section locates the LCE-CL testbed into a two dimensions taxonomy: according to the OSI-model layer where the integration takes place and considering the common and independent processes in the architecture.

\subsection{Integration Models for Different Layers}

There are several architectures using multiple RATs, the basic models - considering the integration layer - are shown in Figure 1 [34]. The LCE-CL testbed integrates disparate access networks using a core IP-layer to manage networking (i.e. heterogeneous networks model).
A. Tunnelled Networks: Upper layers access the different technologies independently. According to some policy, the best network is selected and the Integration Layer tunnels the traffic across the Internet and the chosen RAT. Thus, no modifications are required to the existing network stacks, however, service latency increments, mainly because of functionality duplication and lack of integration in the lower layers.

B. Hybrid Networks: In this model, the individual RATs implement the three bottom layers (Physical, Link, and Network layers). There is a hybrid core that interfaces between the Internet and the different wireless access networks. The main drawback of this model is that networking activities are duplicated, however, the stack does not need to be modified. Nevertheless the service latency reduces because there is not as much redundancy in functionality as in tunnelled networks.

C. Heterogeneous Networks: In this model there is a core layer that deals with all network functionality and operates as a single network to the upper layers. Thus, different RATs implement only the Physical and Link layers, which are specifically related to each technology. A major obstacle of this model is that the different access networks must converge, which requires a huge standardisation effort and operator's commitment.

However, heterogeneous networks are a promising solution for $4 \mathrm{G}$ systems. The integration can be based on Mobile IP, as a protocol used for mobility management issues, with certain modifications to reduce service latency. Thus, modifying the current protocols is not completely unrealistic, it can follow a module-based design to minimise impact to the current stack. 


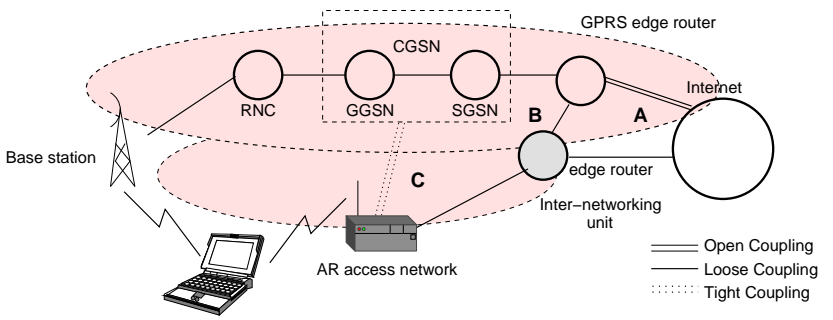

Figure 2. Integration Models: Depending on the integration components.

\subsection{Network Layer Integration Models}

Inter-networking between wireless technologies was considered by the 3GPP TSG [1] working group. This group drafted a feasibility study where they presented four levels of integration between RATs, according to the component where coupling takes place [10]. The main integration scenarios are shown in Figure 2 and listed below.

A. Open Coupling: There is no real integration effort between two or more access technologies. Thus, separated sub-processes take place, however the billing system is shared between networks. These models do not enable seamless inter-technology handovers, a session is terminated when a change in the used RAT being used occurs.

B. Loose Coupling: It is defined as the utilisation of a generic RAT (e.g., WLAN) as an access network complementary to current $3 \mathrm{G}$ access networks. It uses a common subscriber database without any user plane $I u$ interface ${ }^{1}$, i.e. avoiding the SGSN, GGSN nodes. Thus, the RATs are integrated in the network layer by adding special purpose inter-networking components.

C. Tight Coupling: The key characteristic of this model is that the generic access networks (e.g., WLAN) are connected to the core network (e.g., GSM/GPRS) sharing the $I u$ interfaces. Thus, the level of integration impacts the core components - the GGSN and SGSN for the case of Vodafone's GPRS architecture. This enables the integration of most of the operational capabilities into one integrated platform.

There is one more level of integration: fully integrated, it has the same drawbacks as tight coupling, but the integration takes place in core components of both networks.

Broadly, integration architectures have been classified

\footnotetext{
${ }^{1}$ The $I u$ interface provides connection between the Radio Network Controllers (RNCs) and some core nodes in the GPRS network
}

into loose coupling and tight coupling, as the main difference between these two and open coupling is the capability to offer service continuity ${ }^{2}$. The LCE-CL testbed proposes a loose coupling architecture, which enables service continuity -between access and core networks - without affecting the components in the core network.

\section{LCE-CL Testbed Architecture}

To emulate the next generation (4G) integrated networking environment, our experimental testbed setup consists of a loosely-coupled, Mobile IPv6-based GPRS-WLAN-LAN testbed as shown in Figure 4. The cellular GPRS network infrastructure currently in use is the Vodafone UK's production GPRS network. The WLAN access points (APs) are IEEE $802.11 \mathrm{~b}$ APs. Our testbed has been operational since March 2003, and results showing how we optimise vertical handovers are detailed in [13].

In the testbed, the GPRS infrastructure comprises base stations (BSs) that are linked to the SGSN (Serving GPRS Support Node) which is then connected to a GGSN (Gateway GPRS Support node). In the current Vodafone configuration, both SGSN and GGSN node is co-located in a single CGSN (Combined GPRS Support Node). A well provisioned virtual private network (VPN) connects the Lab network to that of the Vodafone's backbone via an IPSec tunnel over the public Internet. A separate "operator-type" RADIUS server is provisioned to authenticate GPRS mobile users/terminals and also assign IP addresses.

For access to the $4 \mathrm{G}$ integrated network, mobile nodes (e.g., laptops) connect to the local WLAN network and simultaneously to GPRS via a Phone/PCCard modem. The Mobile Node's MIPv6 implementation is based on that developed by the MediaPoli project [26], chosen for its completeness and open source nature. We brokered a semi-permanent IPv6 subnet from BTExact's IPv6 Network, which connects us to the 6BONE. Using this address space, we are able to allocate static IPv6 addresses to all our IPv6 enabled mobile nodes. A router in the lab acts an IPv6/IPv4 tunnel end-point to the BTExact's IPv6 network. This router is also an IPv6 access router for the lab's fixed-internal IPv6-enabled network and also for internal WLANs. Routing in the Lab has been configured such that all GPRS/WLAN user traffic going to and from mobile clients are allowed to pass through the internal router, enabling us to perform traffic monitoring.

Since the GPRS cellular network currently operates only on IPv4, We use a SIT (Simple Internet Translation) to tunnel all IPv6 packets as IPv4 packets between the Mobile Node and a machine providing IPv6-enabled access router functionality on behalf of the GPRS network. Ideally, the

\footnotetext{
${ }^{2}$ Service Continuity: services will survive the process of changing access network technology [10]
} 


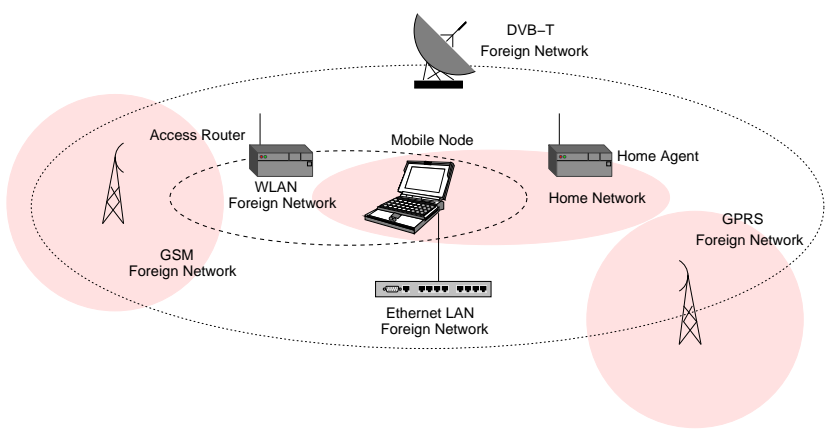

Figure 3. LCE-CL testbed and MIPv6 entities.

GGSN in the GPRS network would provide this functionality directly, but using the tunnel causes only minor overhead, and it represents the current status of IPv4-to-IPv6 migration.

The testbed integrates several independent IP networks, including three IEEE 802.11b sub-networks, Vodafone's GSM/GPRS network, and LCE's local area network. This setup allows us to do experimental analysis of intra-network and inter-network handovers - also known as horizontal and vertical handovers. Figure 3 illustrates how a multi-mode device seamlessly changes its access point between two different radio access technologies (i.e. it performs a vertical handover).

The Mobile Node's home agent provides network connectivity through an IEEE $802.11 \mathrm{~b}$ AP. Additionally, foreign networks (e.g., IEEE $802.11 \mathrm{~b}$ sub-networks, GSM/GPRS Vodafone's network, DVB-T link, and Ethernet LAN) allow the Mobile Node to stay connected by the HA emitting Router Advertisements (RA) and providing a secondary radio coverage. This experimental testbed enables the Mobile Node to perform seamless roaming between heterogeneous technologies, and maintain connectivity with its correspondent nodes.

\subsection{Heterogeneous Technologies}

As mentioned, the testbed integrates heterogeneous wireless technologies with a common IP-layer (i.e. Network Layer). However, below this layer each radio access technology has an independent protocol stack and disparate network characteristics.

Ethernet Connectivity: The Mobile Node is equipped with a IEEE 802.3 network card and supports IPv4 and IPv6 stacks. The Ethernet connection is established through the LCE local network using a cable link. This overlay enables static high-speed networking (10-100Mbps) with small RTTs (1ms).

WLAN Connectivity: As part of the LCE-CL testbed, there are three IEEE $802.11 \mathrm{~b}$ sub-networks, exclusively supporting IPv6. These sub-networks are inter-connected forming a private LCE-IPv6 local network. The LCE-IPv6 network includes the gateway that connects the testbed to 6BONE [11]. The Mobile Node is equipped with WLAN cards and the corresponding network profiles to connect to any of these sub-networks. This overlay enables the Mobile Node to connect using up to $11 \mathrm{Mbps}$, with RTTs as low as $10 \mathrm{~ms}$, and medium mobility.

GSM Connectivity: The Mobile Node has three different GSM phones to connect to the Vodafone's network: a Nokia D211 GSM/GPRS card phone, a Sierra wireless AirCard750, and a Motorola T260 - the main purpose was to evaluate different hardware providers. The Mobile Node connects to the Vodafone network via serial port (for the Motorola T260) or via PCI slot to establish a permanent connection using PPP (Point-to-Point Protocol). This overlay enables voice connection with medium mobility.

GPRS Connectivity: To enable a low-speed data connection (39.6kbps), the Mobile Node has three GPRS radio access devices, which are able to connect to the Vodafone IPv4 GPRS network using a SIT tunnel. The three phones can establish a connection " $3+1^{\prime \prime}$, which is a maximum of 39.6kbps downlink data rate. The Mobile Node establishes a PPP connection, and the user is authenticated in the local RADIUS server. Finally, using the SIT tunnel to encapsulated IPv6 packets into IPv4 packets, it connects to the GPRS network. This overlay enables low-speed, medium mobility data connection with RTTs around $800 \mathrm{~ms}$.

\section{Experimental Setup}

The LCE-CL testbed is formed by 6 workstations, two PDAs, and one laptop, which fulfil different network functionality. Figure 3 shows a MN-centric view of the network architecture. Mobile nodes can connect to the testbed using $100 \mathrm{Mbps}$ Ethernet LAN, WLAN, and the live Vodafone's GSM/GPRS network. Furthermore, we performed experiments with three types of mobile devices: a workstation, a laptop, and the PDAs ${ }^{3}$ (see Table 2).

The connection to the GSM/GPRS cellular network is enabled using a dedicated Access Point Name (APN) located in the Computer Laboratory. The GPRS traffic from Vodafone's network is duplicated and forwarded into the dedicated APN - this enables more accurate measurements, due to real traffic conditions in the GPRS RAT.

IP traffic generated in the mobile nodes is sent over a SIT tunnel between the APN and the Mobile Node due to the lack of support for IPv6 in the Vodafone's network.

\footnotetext{
${ }^{3}$ The PDA has only one PCMCIA slot, it is not possible to have the three interfaces working simultaneously.
} 


\begin{tabular}{|l|l|l|l|}
\hline & Mobile Node \#1 & Mobile Node \#2 & Mobile Node \#3 \\
\hline \hline Device & Workstation & Laptop & PDA \\
\hline Networking role & Multi-mode MN & Multi-mode MN & Multi-mode MN \\
\hline Software & Red Hat 7.1 & Red Hat 7.1 & Linux 2.4.16 \\
& Linux 2.4.16 & Linux 2.4.16 & MIPL 0.9.1 \\
& MIPL 0.9.1 & MIPL 0.9.1 & \\
\hline Network devices & GSM/GPRS, WLAN, & GSM/GPRS, WLAN, & GSM/GPRS, WLAN \\
& LAN & LAN & \\
\hline Hardware & Pentium-II 300MHz & Pentium-III 600MHz & StrongARM II \\
& RAM 364MB & RAM 356MB & RAM 64MB \\
\hline \multicolumn{3}{|l|}{} \\
\hline
\end{tabular}

Table 2. The LCE-CL testbed enables three multi-mode devices.

Ideally, the GGSN will cover the access router functionality directly, with no encapsulation. By using a SIT tunnel from source to destination and implementing the appropriate rules, the problems with firewalls placed in the path between the mobile nodes and the CGSN are overcome otherwise IP traffic will be filtered.

The LCE-CL testbed's Home Agent is a PC with a Pentium-MMX $233 \mathrm{MHz}$ processor and $128 \mathrm{MB}$ in RAM, running Linux 2.4.16 as the operating system, with Red Hat 7.1 and MIPL 0.9.1 distributions.

The Access Router for the live GPRS network - and the end point of the SIT tunnel - is a PC connected to the CGSN via an IPsec VPN, it has a Pentium-IV $1500 \mathrm{MHz}$ processor and 512MB in RAM. The operating system is Linux 2.4.16 with Red Hat 7.1 distribution.

The last network device to complete a MIPv6-based scenario is the Correspondent Node. It is a Pentium-III $800 \mathrm{MHz}$ with $128 \mathrm{MB}$ in RAM, running Linux 2.4 .16 with Red Hat 7.1 and MIPL 0.9.1 distributions. It is important to notice that all the LCE-CL network components are IPv6enabled.

\subsection{LCE-CL Testbed Network-Centric View}

Figure 4 shows a network-centric view of the testbed. The LCE-CL enables horizontal and vertical handovers through the integration of seven networks based on different technologies. There are three WLANs, one functioning as Home Network and the other two as Foreign Networks (i.e. Visited Networks), which enable homogeneous handovers.

Furthermore, an IPv6-LAN allows the interconnection of the WLANs and mobile nodes to the IPv6 backbone (i.e. 6BONE), and it enables wired-to-wireless handovers (functioning as a foreign network). Finally, the IPv4-based LCE local network connected to the Vodafone's network allows access to the live GPRS RAT, completing the testbed - on which wired-to-wireless and cellular-to-hotspots roaming is possible.

The fully-integrated LCE-CL testbed allows experimental work emulating next generation Communication systems in multiple scenarios. Section 7 shows the software used to implement node mobility on the testbed. Furthermore, these distributions were modified to fulfil particular requirements, the main changes are mentioned in that section, as well.

\section{Software Distributions}

This section describes the main software distributions used in the experimental setup, as well as the most relevant modifications to the code.

\subsection{Mobile IPv6 modified version}

A major justification to start this project was to motivate IPv6 deployment and investigate the drawbacks and strengths of migrating IPv4. In this context, we decided to use Mobile IPv6 because it is based on IPv6 and it has advantages over its predecessor (Mobile IPv4) such as explicit mobility headers, address size, and eliminates the use of foreign agents. Furthermore, we needed an open environment to achieve modifications to the distribution and to develop new components.

Thus, to enable terminal mobility in the LCE-CL testbed, we used MIPL [26] as a Linux implementation of the Internet draft for Mobile IPv6 mobility support [20] - this last version of the draft became RFC 3775 in June 2004 [21]. Based on this distribution, we did the following modifications to the source code:

- Added support for Binding Update bi-casting: The Mobile Node's code was changed, particularly the send-binding-update and send-options routines.

- Added support for soft-handovers: Mobile Node's functionality was modified to accept packets with the old interface IP address as the destination address, while performing the registration with the new radio access technology.

Apart from MIPL, there is only one more implementation of Mobile IPv6 for Linux. Lancaster University has the oldest implementation, however, they stopped supporting this distribution in 1998. Thus, the last kernel supported was 2.1.9 and it is compatible with IETF Mobile IPv6 draftv5 (last version of this draft was 24).

\subsection{RADVD modified version}

The Linux IPv6 Router Advertisement Daemon (radvd) send Router Advertisements, specified by RFC 2461 to the specified interfaces, according to the configuration parameters or as a response to a Router Solicitation. These messages are required to support IPv6 stateless autoconfiguration. The version used in the testbed is radvd 0.9.1 with some modifications: 


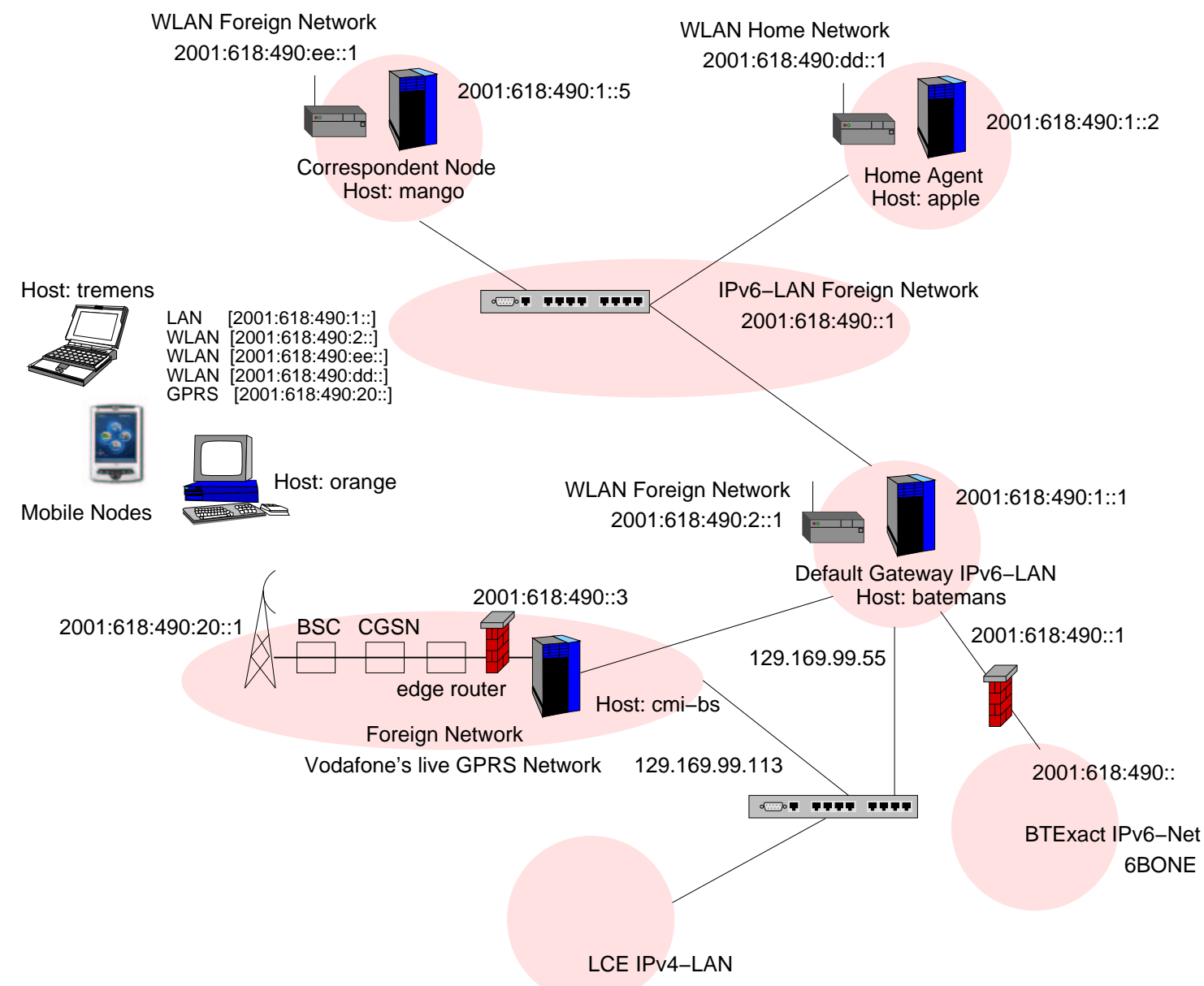

Figure 4. Network-centric view of the LCE-CL testbed.

- Added support to change Router Advertisement frequencies, setting values above the ones recommended by RFC 3775 (MIPv6).

- Added support to send hints to user space, specially to a mobility support middleware developed in the Laboratory for Communication Engineering [33].

\subsection{Other software components}

Additional software was used to perform the mentioned experiments:

MGEN [6] was used to generate UDP/IPv6 traffic and perform trace analysis to calculate raw Network Layer MIPv6 latency.

TRPR [9] was used to review the collected traces and produce suitable plots to show the results.

TCPDUMP [7]. The traffic passes through an intermediate router, and using tcpdump it is collected for fur- ther analysis. The collected protocols are: UDP/IPv6, TCP/IPv6, and ICMPv6.

TCPTRACE [8]. Transport level plots were generated using tcptrace. Since the latest version of tcptrace for analysing TCP traces does not offer any support for MIPv6, we have also modified it to handle Mobile IPv6 connections.

\section{Experimental Results}

This section describes some experiments performed using the LCE-CL testbed and the off-the-shelf version of MIPL (non-optimised). The motivation of these research is to study how MIPv6 behaves in real heterogeneous networks under real conditions. This allows us to identify the problems posed by $4 \mathrm{G}$ networks.

The first experiments were focused on detecting MIPv6 overhead in different levels of the protocol stack: network, transport and application layers, with the intention to propose methods to improve overall performance. 


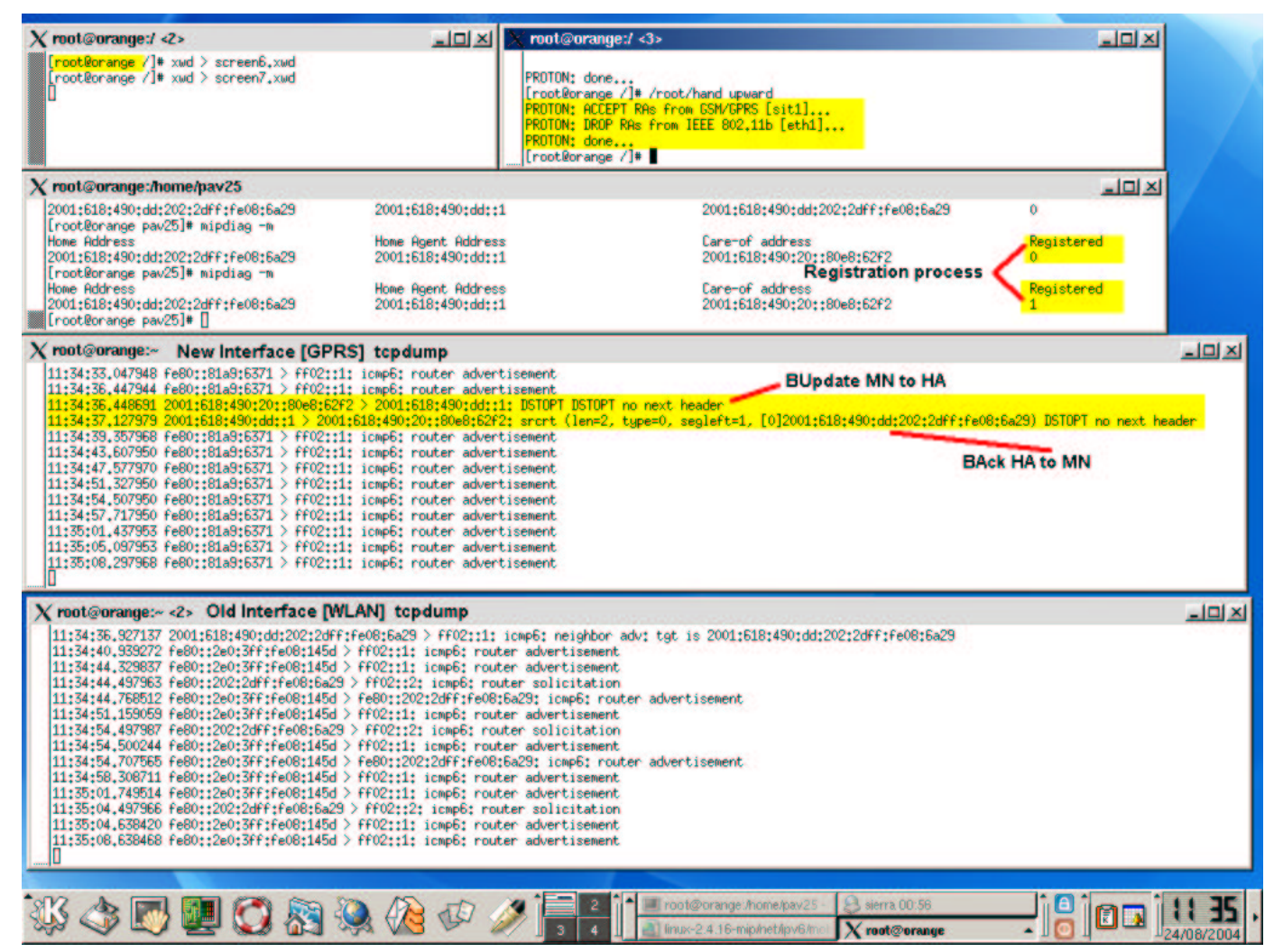

Figure 5. Mobile node's output when performing an inter-technology handover.

\subsection{Mobile IPv6 network layer performance}

To calculate the latency in the network layer based on packet loss, we generate ICMPv6 traffic between the Correspondent Node and the Mobile Node. The Correspondent Node sends small ICMPv6 packets (104 bytes) at a high configurable rate (every $200 \mathrm{~ms}$ ). Thus, the handover latency is the product of the number of packets lost multiplied by the time interval between the packets (see Figure 6).

Results shown in Figure 6 suggest that Mobile IPv6 protocol was designed for mobility management within the same technology. When dealing with heterogeneous handovers, MIPv6 does not perform as expected and in most scenarios latency exceeds acceptable limits (not even close to support real-time applications). We should mention that the presented values can have a precision error of $200 \mathrm{~ms}$ due to the experimental setup, thus handover latency from IEEE $802.11 \mathrm{~b}$ to GPRS is between $2200 \mathrm{~ms}$ and $2400 \mathrm{~ms}$. In fact, the mean handover latency for this scenario is $2325 \mathrm{~ms}$.

\subsection{Mobile IPv6 transport layer performance}

In addition to study MIPv6 performance at network layer level, it is also interesting to investigate how current transport protocols affect mobility management in heteroge- neous environments. This section discusses related results collected during some experiments that were done in the LCE-CL testbed (Figure 5 shows the console at the Mobile Node during the experiments).

Several tests were done using MGEN in order to analyse UDP effects on MIPv6. During the experiments, the receiver (i.e. Mobile Node) collects transmitted packets and creates $\log$, which are then analysed to extract statistical data such as perceived latency in the Mobile Node.

Figure 7 shows the latency at the Mobile Node. In this scenario, the Correspondent Node (CN) starts sending UDP packets (packet size 80 bytes, every $50 \mathrm{~ms}$ ). After 22s we can observe that a handover occurred, and 57 packets were lost - this implies a handover latency of $2850 \mathrm{~ms}$. TCP related experiments have also been performed. Some results can be found in [13].

\section{Conclusions}

We believe that experimental environments represent an essential tool in the deployment of future mobile networks. In this paper, we described a loosely-coupled testbed that can be considered as a trial for $4 \mathrm{G}$ Systems. 


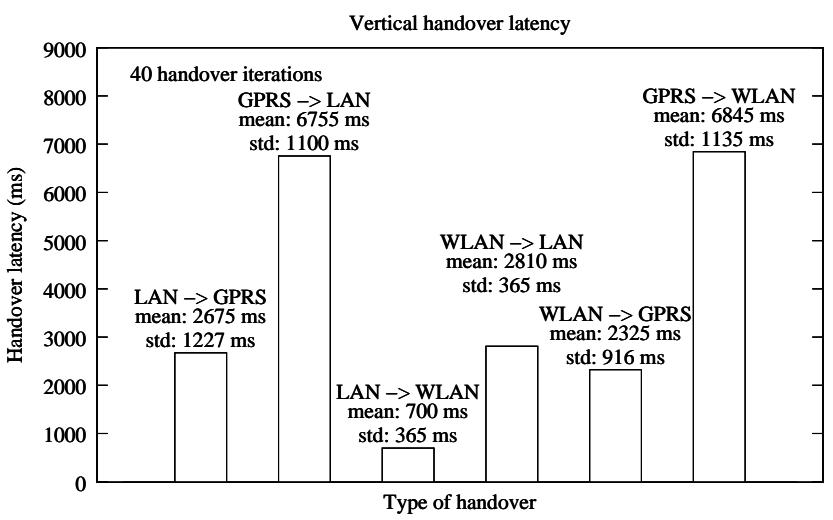

Figure 6. MIPv6 Layer 3 vertical handover latency.

The main points that distinguish our testbed from other setups are that (1) it fully evaluates MIPv6 in heterogeneous environments, while other projects focus on the evaluation of intra-technology handovers using MIPv6 or intertechnology handovers using MIPv4 due to constraints in the architecture, and (2) Vodafone's GSM/GPRS network. As far as we know, our testbed is the only one that enables a GSM/GPRS overlay using the actual provider's network (i.e. Vodafone). Other projects emulate a GPRS link or install an isolated GPRS network for experimental purposes, both cases lack real-life conditions.

The testbed is used as the platform for unveiling, analysing, solving, developing, and testing the following technical issues:

- Seamless horizontal handovers using MIPv6

- MIPv6 performance during vertical handovers

- A client-based solution to improve performance in horizontal handovers for WLANs [27]

- Methods to minimise vertical handover latency

- Policy-based solution to manage multiple interfaces

- Policy-based solution to provide full mobility support

- QoS-based handover algorithms for overlay networks

- Context aware algorithms for overlay networks

Finally, we showed the value of this setup by including experiments and discussing the importance of deploying real systems to unveil problems and evaluate potential solutions.

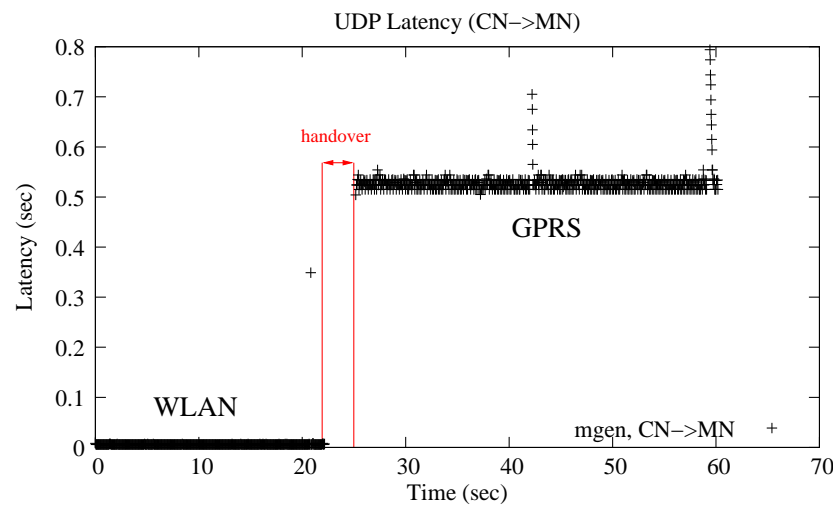

Figure 7. MIPv6 UDP vertical handover latency.

\section{Acknowledgements}

The authors would like to thank Professor Andy Hopper for his essential support to this project. Also, thanks to Calicrates Policroniades, Javier Baliosian, Eleftheria Katsiri, and Rajiv Chakravorty for their helpful comments. Pablo Vidales has a scholarship from the Mexican Government through the National Council of Science and Technology (CONACyT). Carlos Jesús Bernardos is partially sponsored by the European Union under the E-Next Project FP6506869 .

\section{References}

[1] 3rd Generation Partnership Project, Technical Specification Group Services and System Aspects. http: / /www. 3gpp.org/TB/home.htm.

[2] EU FP6 Daidalos project. http://www.ist-daidalos.org.

[3] EURESCOM. http://www.eurescom.de.

[4] IST-MIND (Mobile IP based Network Developments) project. http: //www. ist-mind.org.

[5] IST-NOMAD project. http://www . ist-mind.net.

[6] MGEN. The Multi-Generator Toolset. http://mgen.pf.itd.nrl.navy.mil/.

[7] TCPDUMP. http://www.tcpdump.org.

[8] TCPTRACE. http://www.tcptrace.org.

[9] TRPR. TRace Plot Real-time. http://proteantools.pf.itd.nrl.navy.mil/trpr.html.

[10] 3GPP TSG/WG. Feasibility study on 3GPP system to Wireless Local Area Network (WLAN) interworking (Release 6), 2003.

[11] 6BONE. Testbed for the deployment of IPv6.

[12] M. Buddhikot, G. Chandranmenon, S. Han, Y. W. Lee, S. Miller, and L. Salgarelli. Integration of 802.11 and thirdgeneration wireless data networks. In Proceedings of IEEE INFOCOM 2003, March 2003. 
[13] R. Chakravorty, P. Vidales, K. Subramanian, I. Pratt, and J. Crowcoft. Performance issues with vertical handovers experiences from gprs cellular and wlan hot-spots integration. In Proceedings of The Second IEEE International Conference on Pervasive Computing and Communications (PerCom'04), March 2004.

[14] A. Cuevas, P. Serrano, C. J. Bernardos, J. I. Moreno, J. Jaehnert, K. Hyung-Woo, J. Zhou, D. Gomes, P. Gonçalves, and R. Aguiar. Field Evaluation of a 4G True-IP network. In IST Mobile Summit 2004, 2004. Lyon, France.

[15] N. A. Fikouras, A. Udugama, C. Görg, W. Zirwas, and J. M. Eichinger. Experimental Evaluation of Load Balancing for Mobile Internet Real-Time Communications. In Proceedings of the Sixth International Symposium on Wireless Personal Multimedia Communications (WPMC), YokosukaKanagawa, Japan, October 2003.

[16] FIT-MIP(a). Definition of terminology for Mobile IP Definition of IP services in the Mobility Context, 2003. http: / / www . eurescom.de/.

[17] FIT-MIP(b). First steps towards UMTS: Mobile IP services, an European testbed, 2003. http: //www . eurescom. de/.

[18] E. García and P. R. et al. MIND Trials Final Report. Project Deliverable, November 2002.

[19] ISI-USC. Transmission Control Protocol and Internet Protocol specifications, IETF RFC 791 and IETF RFC 793, 1981.

[20] D. B. Johnson, C. E. Perkins, and J. Arkko. Mobility support in IPv6 (draft-ieft-mobileip-ipv6-24.txt) http: //www. ieft.org, 2003.

[21] D. B. Johnson, C. E. Perkins, and J. Arkko. Mobility Support in IPv6 (RFC 3775) http: / / www . ieft. org, June 2004.

[22] R. H. Katz. Adaptation and mobility in wireless information systems. IEEE Personal Communications, 1:6-17, 1994.

[23] K. Kuladinithi, A. Konsgen, S. Aust, and N. A. Fikouras. Mobility Management for an Integrated Network Platform. In Proceedings of the Fourth IEEE Conference on Mobile and Wireless Communication Networks (MWCN 2002), Stockholm, Sweden, September 2002.

[24] K. Lai, M. Roussopoulos, D. Tang, X. Zhao, and M. Baker. Experiences with a mobile testbed. In Proceedings of The Second International Conference on Worldwide Computing and its Applications (WWCA'98), March 1998.

[25] V. Marques, R. Aguiar, C. Garcia, J. Moreno, C. Beaujean, E. Melin, and M. Liebsch. An IP-based QoS architecture for $4 \mathrm{G}$ operator scenarios. IEEE Wireless Communications Magazine, June 2003.

[26] MIPL. Mobile IP for Linux (MIPL). Developed by HUT Laboratory for Theoretical Computer Science - GO/Core project http: / /www. mobile-ipv6. org.

[27] L. Patanapongpibul and G. Mapp. A Client-based Handoff Mechanism for Mobile IPv6 Wireless Networks. In Proceedings of the 8th IEEE Symposium on Computers and Communications (ISCC), Kiris-Kemer, Turkey, July 2003.

[28] C. E. Perkins. IP Mobility Support for IPv4 (RFC 3344) http: //www. ieft.org, 2002.
[29] P. Philippopoulos, P. Fournogerakis, I. Fikouras, N. Fikouras, and C. Görg. NOMAD: Integrated Networks for Seamless and Transparent Service Discovery. In Proceedings of the IST Mobile Summit 2002, 2002.

[30] P. Ruiz, E. Mitjana, and L. Burness. Advanced services over future wireless and mobile networks in the framework of the MIND project. In Proceedings of the Terena Networking Conference (TNC2002), Limerik, Ireland, June 2002.

[31] A. Sanmateu, L. Morand, E. Bustos, S. Tessier, F. Paint, and A. Sollund. Using mobile IP for provision of seamless handoff between heterogeneous access networks, or how a network can support the always-on concept. EURESCOM Summit, 2001. http: //www . eurescom.de/.

[32] M. Stemm and R. H. Katz. Vertical handoffs in wireless overlay networks. Mobile Networks and Applications, 3(4):335-350, 1998.

[33] P. Vidales, R. Chakravorty, and C. Policroniades. PROTON: A Policy-based Solution for Future $4 \mathrm{G}$ devices. In Proceedings of 5th International Workshop on Policies for Distributed Systems and Networks (IEEE POLICY 2004), New York, United States, June 2004.

[34] G. Wu, P. Havinga, and M. Mizuno. Wireless Internet on Heterogeneous Networks. In Proceedings of the IEEE Global Telecommunications Conference (GLOBECOM 2001), San Antonio, US, November 2001.

[35] M. Ylianttila, M. Pande, J. Makela, and P. Mahonen. Optimization scheme for mobile users performing vertical handoffs between IEEE 802.11 and GPRS/EDGE networks. In Proceedings of the 13th IEEE International Symposium on Personal, Indoor and Mobile Radio Communications, September 2002. 